\title{
Uma Metodologia de Projeto para Reconfiguração Parcial e Dinâmica em Aplicações de Fog-Computing
}

\author{
Iuri S. Castro* Janier Arias-Garcia ${ }^{* *}$ Alair Dias Junior* \\ * Programa de Pós-Graduação em Engenharia Elétrica, Universidade \\ Federal de Minas Gerais, MG, 31270-901, Brasil, \\ e-mail: iuric,alairjunior@ufmg.br \\ ** Departamento de Engenharia Eletrônica, Universidade Federal de \\ Minas Gerais, MG, 31270-901, Brasil, \\ e-mail: janier-arias@ufmg.br
}

\begin{abstract}
Cloud computing has been increasingly used in industrial environments. This decentralized computing architecture promotes cost reduction, reduces the time-to-market and improves flexibility. However, a common side-effect of this approach is an increase in latency. Fog-computing is an ideal solution to this latency-sensitive applications as it allows the edgedevices to carry out a substantial amount of computation locally. Reconfigurable architectures implemented in SoC-FPGAs meet those requirements, especially when Dynamic and Partial Reconfiguration (DPR) is used. Using DPR is challenging, however, as architectural design can be complex without supporting methodologies and architectural models. This work presents a methodology and a supporting architectural model for the application of Dynamic and Partial Reconfiguration in SoC-FPGAs targeting fog-computing applications to the industry 4.0. The methodology and architecture proposed have been applied with success to an illustrative example.

Resumo: Recentemente, observa-se um crescimento da utilização de computação em nuvem na indústria. Esse modelo permite a descentralização da infraestrutura, reduzindo custos, diminuindo o time-to-market (TTM) e aumentando a flexibilidade. No entanto, um efeito colateral dessa abordagem é o aumento da latência. Para sistemas sensíveis à latência, fogcomputing se apresenta como uma solução ideal, pois parte da computação é realizada nos dispositivos de fronteira. Arquiteturas reconfiguráveis implementadas em SoC-FPGAs mostramse eficientes para atender tais requisitos, especialmente quando empregada reconfiguração parcial e dinâmica. Uma dificuldade dessa abordagem é a complexidade do projeto que requer um suporte de metodologias e modelos de arquitetura adequados. Nesse trabalho apresenta-se uma proposta de arquitetura e de metodologia para aplicar a técnica de reconfiguração parcial e dinâmica em plataformas SoC-FPGAs em aplicações de fog-computing para indústria 4.0. A metodologia e arquitetura proposta são aplicadas com sucesso em um exemplo ilustrativo.
\end{abstract}

Keywords: Palavras-chaves: Dynamic and Partial Reconfiguration; FPGA; Hardware Accelerators; Design flow, Fog-computing.

Palavras-chaves: Reconfiguração Parcial e Dinâmica; FPGA; Aceleradores via Hardware; Fluxo de Projeto, Fog-computing.

\section{INTRODUÇÃO}

Com o avanço tecnológico dos últimos anos, a demanda por poder computacional em sistemas embarcados aumenta. Aplicações que envolvem, por exemplo, visão computacional e inteligência artificial, tornam-se comuns em ambientes industriais e domésticos onde as limitações de projeto impostas a essas aplicações, tais como área em silício, custo, precisão, consumo, robustez, entre outras, tornam o projeto desses sistemas cada vez mais desafiador (Krill et al., 2010; Nava et al., 2011; Claus et al., 2007).

Recentemente, observa-se uma crescente tendência no emprego de computação em nuvem em diferentes contextos.
Nesse paradigma, desafios importantes são impostos aos projetistas, como por exemplo lidar com alocação de recursos sob demanda. Mesmo a computação em nuvem sendo tão amplamente empregada, nota-se que diversos problemas ainda são tópicos importantes de pesquisa. Um deles é aumentar o desempenho e eficiência da comunicação entre a nuvem e os chamados end devices, visando a implementação de sistemas sensíveis à latência (Mouradian et al., 2018). Diversas aplicações que requerem análise em tempo real de dados provenientes de sensores complexos, como é o caso das câmeras, são incluídas nessa categoria, sendo exemplos o monitoramento de qualidade de produtos por meio de visão computacional, aplicações para detecção 
e combate a incêndios e veículos conectados, além das redes inteligentes (Nguyen and C. Hoe, 2018).

Esse crescimento do emprego da computação em nuvem traz consigo uma exigência por novas tecnologias que permitam o desenvolvimento de aplicações em ambientes industrias. Contudo, o projeto de sistemas embarcados, onde o time-to-market (TTM) é um fator crucial para sucesso e onde alto poder de processamento é cada vez mais necessário, nem sempre consegue atender todas as exigências determinadas durante a fase de levantamento de requisitos, em especial devido ao trade-off entre os diferentes parâmetros de projeto (Vahid and Givargis, 2001).

Nesse contexto, atualmente o projetista de sistemas embarcados enfrenta dificuldades quanto às tecnologias disponíveis para a implementação do seu projeto em sistemas embarcados, uma vez que suas decisões de projeto normalmente se limitam a buscar por uma plataforma de desenvolvimento comercial que atenda às especificações. Arquiteturas reconfiguráveis, por outro lado, podem atender às necessidades específicas de um dado projeto (Choi, 2011). Por meio de um fluxo de projeto robusto e consistente, elas permitem combinar alto desempenho e baixo consumo, sem sacrificar a flexibilidade, obtendo-se uma maior generalização de desenvolvimento das plataformas propiciando uma diluição do custo de projeto entre várias aplicações e uma redução do TTM. Estas vantagens se tornam ainda mais acentuadas quando se utiliza as chamadas SoC-FPGAs, que combinam o desempenho superior dos ASICs (Application Specific Integrated Circuits) com o TTM reduzido dos sistemas baseados em microcontroladores, por oferecerem um ou mais processadores combinados a uma rede de lógica reconfigurável em um único SoC (System-on-Chip).

Com base nessa mesma ideia, uma das vantagens pouco conhecida dos sistemas reconfiguráveis do tipo FPGA (Field-Programmable Gate Array), é a técnica de Reconfiguração Parcial e Dinâmica (Dynamic Partial Reconfiguration, DPR) que permite a reconfiguração de partes do FPGA em tempo de execução, sem corromper ou afetar o funcionamento das outras partes que possam estar executando (Xilinx, 2018), possibilitando a reutilização e multiplexação por tempo de recursos de hardware, reduzindo a área e potência dissipada pelo sistema como um todo (Kareemullah et al., 2017; Lie and Feng-yan, 2009; Krill et al., 2010). A técnica permite também um ganho de escalabilidade do sistema, onde novas funcionalidades podem ser posteriormente implementadas e inseridas em um sistema testado e operacional.

Uma das dificuldades do uso dos sistemas reconfiguráveis tem relação com seu fluxo de desenvolvimento. Enquanto que nos sistemas micro-controlados faz se necessário um conhecimento de linguagens de alto nível $(\mathrm{C} / \mathrm{C}++$ na maioria dos casos), em hardware reconfigurável há necessidade de se ter um conhecimento de linguagens de descrição de hardware, assim como um grau de entendimento de sistemas digitais, além de maturidade para explorar o paralelismo intrínseco dessas plataformas. Essa dificuldade somada ao nível de complexidade da metodologia no fluxo de projeto das ferramentas tem afastado grande parte da comunidade quanto ao uso de sistemas reconfiguráveis.
Alguns trabalhos buscaram facilitar a integração e desenvolvimento de aplicações utilizando aceleradores através da virtualização do hardware. Kelm and Lumetta (2008) desenvolveram uma extensão para Linux nomeada HybridOS, possibilitando que desenvolvedores de software da aplicação possam fazer chamadas ao hardware através de uma API (Application Programming Interface), tornando transparente toda a implementação do hardware e a comunicação, deixando sob responsabilidade do sistema operacional o escalonamento e compartilhamento dos aceleradores. Similarmente, Vatsolakis and Pnevmatikatos (2017) desenvolveram uma API nomeada RACOS, dando suporte à carga/descarga dos aceleradores, assim como escalonamento e comunicação transparentes ao usuário.

Contudo, métodos e fluxos de projeto que possibilitem a exploração dessas caraterísticas presentes em algumas FPGAs/SoCs ainda são bem escassos e limitadas nas suas informações, dificultando o desenvolvimento sem um nível de conhecimento avançado em hardware digital. Assim, neste trabalho, propõe-se uma metodologia de projeto para guiar o processo de desenvolvimento de sistemas hardwaresoftware utilizando plataformas SoC que possam ter ganhos com a aplicação de DPR. Desta forma, podemos citar como principais contribuições deste trabalho as seguintes:

- Uma metodologia para desenvolvimento de sistemas hardware-software utilizando DPR;

- Apresentação de uma arquitetura minimalista capaz de dar suporte ao emprego de DPR;

- Uma padronização da interface lógica dos aceleradores e uma API para tornar o acesso e controle dos mesmos transparente para o usuário.

O trabalho está organizado da seguinte forma: Seção 2 apresenta a metodologia desenvolvida, apresentando as padronizações feitas e ao final um diagrama de fluxo para a aplicação da metodologia, a Seção 3 mostra a utilização da metodologia proposta em uma aplicação simples a fim de prova de conceito, e a Seção 4 discute as conclusões deste trabalho.

\section{METODOLOGIA PROPOSTA}

A metodologia proposta baseia-se na padronização da interface dos aceleradores, tanto a interface física quanto interface lógica. A padronização da interface física é necessária para que seja aplicada a técnica de reconfiguração parcial e dinâmica (DPR), enquanto a padronização da interface lógica permite que uma API seja capaz de tornar transparente para o usuário a camada de hardware envolvida na reconfiguração parcial.

Então, para dar suporte a metodologia proposta, faz-se a padronização dos aceleradores e define-se uma arquitetura minimalista que servirá de modelo para validação. Assim, nas subseções seguintes apresenta-se a arquitetura de suporte definida (subseção 2.1), a padronização adotada nos aceleradores (subseção 2.2) e o API (subseção 2.3) para integração e transparência do sistema. Ao fim um diagrama de fluxo da metodologia é mostrado (ver Figura 3 ), e suas etapas enumeradas. 


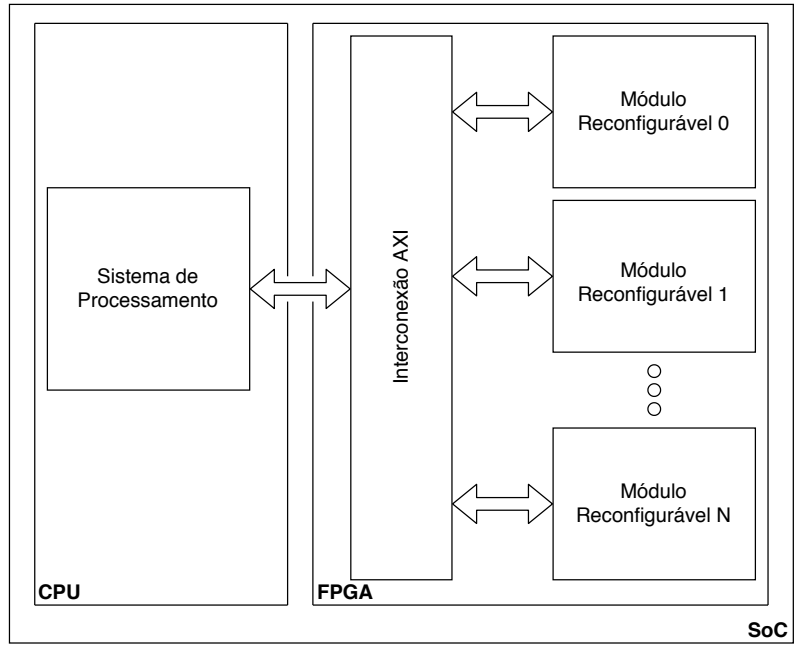

Figura 1. Arquitetura do sistema.

\subsection{Arquitetura}

A definição de uma arquitetura apropriada faz-se necessária para delimitar o desenvolvimento dos aceleradores, e, posteriormente, possibilitar uma interface transparente, por meio da API, para o desenvolvedor do software da aplicação.

Os Módulos Reconfiguráveis (MR) da arquitetura, Figura 1 , são os módulos que poderão ser reconfigurados em tempo de execução, sendo eles os receptáculos dos aceleradores. A interface entre os aceleradores e o Sistema de Processamento (Processing System, PS) é feita utilizando o AMBA AXI4-Lite desenvolvido pela empresa ARM. As interfaces seguem um modo Mestre-Escravo e são conectadas via interconector, de acordo com a especificação do barramento (ARM, 2013). Os módulos implementam, necessariamente, uma interface no modo escravo para se comunicar com o PS. Caso haja aceleradores que necessitem de acesso direto à memória, implementa-se também uma segunda interface no modo mestre. Vale ressaltar que essa é uma arquitetura minimalista para dar suporte aos métodos propostos nesse trabalho, podendo o projetista expandir essa arquitetura de acordo com a necessidade de sua aplicação.

\subsection{Aceleradores}

Assim como a interface física, a interface lógica dos aceleradores também precisa seguir um modelo de desenvolvimento, para que a API consiga identifica-los e comandalos.

A interface AXI4-Lite modo escravo permite que os aceleradores sejam mapeados em memória, possibilitando que sejam controlados através de leituras e escritas em registradores. A Figura 2 permite uma visão geral da padronização adotada para os registradores de um acelerador, onde um modelo com 16 registradores de 32-bits foi definido, com os dois primeiros registradores com funções específicas e os 14 restantes como registradores de uso geral, disponíveis ao desenvolvedor do acelerador.

Os registradores são acessados através de um endereço de memória base e um offset, sendo calculados conforme

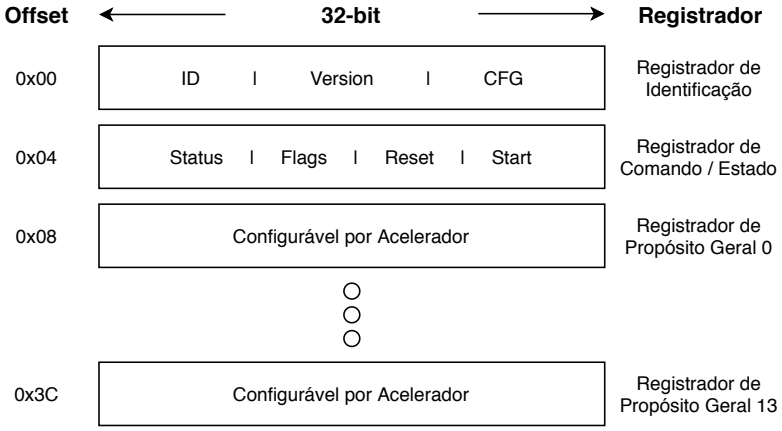

Figura 2. Registradores do acelerador mapeados em memória.

(1). Os endereços de memória base são fixos para cada módulo reconfigurável e definidos durante o projeto da região estática (vide Seção 2.4).

$$
\text { Address }_{\text {reg } N}=\text { BaseAddress }_{\text {accel }}+(N * 4)
$$

Como dito anteriormente, e observando-se a Figura 2, os registradores 0 e 1 são utilizados para identificação e controle por parte da API, sendo:

Registro 0 utilizado para a identificação do acelerador, sendo um registro de apenas leitura. O registrador define a identificação única do acelerador $I D$ em 16-bits, possibilitando até 65536 identificadores de aceleradores diferentes, a versão do acelerador Version em 8-bits, dando suporte a compatibilidade entre diferentes versões de aplicações, e a configuração Config em 8-bits, informando qual é o mapeamento (floorplanning) utilizado pelo acelerador.

Registro 1 utilizado para comandar a execução do acelerador e verificar estado. O registrador define os bits de comando Reset e Start, sendo eles responsáveis por, respectivamente, reiniciar o acelerador em um estado conhecido, limpando condições de erro caso haja, e iniciar execução. É definido também o campo Flag em 4-bits, onde o desenvolvedor pode indicar para a aplicação informações de como foi a execução do acelerador (indicando, por exemplo, qual o código do erro ocorrido), e o campo de Status em 2-bits, podendo ele indicar:

- $\left(00_{2}\right)$ Ready: pronto para executar;

- $\left(01_{2}\right)$ Busy: ocupado executando;

- $\left(10_{2}\right)$ Done: terminou de executar;

- $\left(11_{2}\right)$ Error: erro durante execução.

Os registradores de 2 até 15 ficam a disposição do desenvolvedor do acelerador, podendo ser utilizados para entradas de dados, como: operandos, endereços de memória, quantidade de dados, parâmetros, etc. ou como saída de dados e resultados de operações. Não é necessário implementar todos os registradores caso o acelerador não necessite, porém todos devem ser mapeados.

\subsection{Application Programming Interface (API)}

A ideia principal da API é tornar transparente para o usuário a configuração e reconfiguração dos aceleradores e dar suporte ao acesso de dados e controle. Usuários podem utiliza-lá e criar bibliotecas para os aceleradores 
desenvolvidos, gerando camadas de software acima, assim como é feito em desenvolvimento de periféricos em sistemas micro-controlados, aumentando a reutilização de código.

Implementou-se na API um escalonador do tipo LFU ( $L e$ ast Frenquently Used), para controlar quais aceleradores serão substituídos durante a reconfiguração. O escalonador associa à cada acelerador um contador de utilização, assim, quando instanciado a função de reconfiguração, é verificado qual acelerador possui menor contagem de utilização sendo ele escolhido para ser substituído.

Pode-se, então, destacar as funções básicas implementadas, sendo:

- Inicialização: Configuração da região estática e inicialização do escalonador;

- Listagem de aceleradores: Retorna os aceleradores configurados nas regiões reconfiguráveis, assim como versão e código de configuração de cada um.

- Verificar acelerador: Verifica se um acelerador específico está configurado.

- Configura acelerador: Adiciona o acelerador na lista do escalonador para ser configurado.

- Verificar Estado: Verifica estado de execução do acelerador.

- Escrever em Registrador: Escrever dados em registradores do acelerador.

- Ler Registrador: Ler dados dos registradores do acelerador.

- Iniciar Execução: Indicar ao acelerador para iniciar a execução.

- Reiniciar: Reiniciar acelerador, limpando flags de erro.

- Ler Flags: Retorna as flags de execução do acelerador.

É de responsabilidade do API garantir que durante a reconfiguração dos aceleradores, os mesmos estejam inativos no barramento AXI, ou seja, não estejam fazendo nenhuma transação de dados.

\subsection{Fluxo de Projeto}

O fluxo de projeto indica ao desenvolvedor, de forma clara, quais são as etapas da metodologia proposta e a sequência a ser seguida durante o processo de desenvolvimento. Essa metodologia proposta define 9 etapas que são apresentadas na forma de um fluxograma na Figura 3, sendo:

Definições de Projeto Definições gerais de projeto, como o tamanho da área lógica programável do SoC utilizado, frequência de operação, periféricos, aceleradores de hardware que serão utilizados, memória, entre outros. O tamanho do SoC vai influenciar diretamente a quantidade de módulos reconfiguráveis possíveis e o tamanho máximo dos aceleradores.

Descrição do Acelerador São feitas as implementações dos aceleradores, podendo ser feita em HDL (Hardware Desciption Language) ou em outras linguagens de alto nível, desde que tenha o compilador para transforma-la em HDL. Blocos de IP (Intellectual Property) de terceiros podem ser utilizados para implementar a lógica do acelerador.

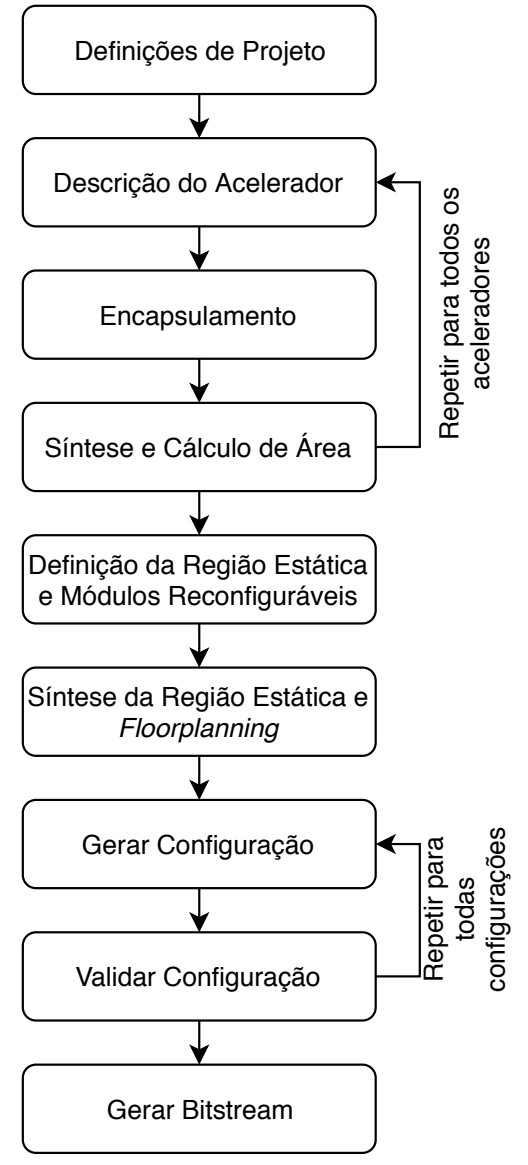

Figura 3. Diagrama de fluxo de projeto.

Encapsulamento A lógica do acelerador descrita é então encapsulada no modelo de acelerador definido, implementando a interface AXI4-Lite Escravo seguindo as definições para os registradores e, para o caso em que o acelerador necessite do acesso direto a memória, é implementado também a interface AXI4 Mestre.

Síntese e Cálculo de Área Faz-se a síntese dos aceleradores no modo fora de contexto (out-of-context), para que não sejam associados blocos de entrada e saída (IO) para o acelerador. A síntese estima a quantidade de recursos utilizado pelo acelerador, sendo esses valores utilizados para especificar o tamanho das regiões dos módulos reconfiguráveis.

Repete-se as etapas Descrição do Acelerador, Encapsulamento e Síntese e Cálculo de Área sequencialmente para cada acelerador.

Definição de Região Estática e Módulos Reconfiguráveis Define-se a região estática do projeto, implementando todos os periféricos fixos e fornecendo a estrutura da arquitetura minimalista apresentada na seção 2.1. A quantidade de módulos reconfiguráveis será definida a partir da quantidade de recursos disponíveis no SoC e do tamanho e tipo de recursos utilizados pelos aceleradores. Geralmente o gargalo estará em aceleradores grandes e na variedade de tipos de recursos necessários (blocos DSP, blocos RAM). São associados aos módulos reconfiguráveis um endereço de memória base para a interface AXI4-Lite Escravo, pois a mesma é mapeada em memória. 
Síntese da Região Estática e Floorplanning O processo de síntese da região estática é dependente da ferramenta de desenvolvimento utilizada. A ferramenta Vivado do fabricante Xilinx trabalha com a síntese da região estática tratando os MR como caixa-pretas, enquanto a ferramenta Quartus do fabricante Intel FPGAs trabalha com um processo de revisões, sendo a região estática a revisão base. Ao contrário de um projeto comum, onde o floorplanning é utilizado apenas por peritos para otimizar o uso de área e performance, o projeto envolvendo DPR necessita que o desenvolvedor defina manualmente no chip as regiões dos MR. O desenvolvedor deve-se atentar à arquitetura do dispositivo utilizado, pois os dispositivos atuais possuem arquiteturas heterogêneas, com os seus recursos como blocos de DSP e RAM espalhados de forma não homogenia. Devese verificar também as limitações impostas pela ferramenta e a arquitetura do chip.

Gerar Configuração É considerado como configuração a implementação da região estática e um acelerador ocupando um dos MRs. A configuração é então gerada substituindo o acelerador sintetizado em um dos MR que o atende, fazendo então o roteamento do mesmo.

Validar Configuração As configurações são validadas entre si, ou seja, são comparadas umas com as outras, verificando se o roteamento e a posição da região estática são equivalentes, assim como as interfaces dos aceleradores. Repete-se as etapas de Gerar Configuração e Validar Configuração para cada configuração desejada, ou seja, para cada acelerador em cada módulo reconfigurável.

Gerar Bitstream Geram-se as bitstreams totais, contendo a região estática, e as parciais, contendo apenas o acelerador implementado no MR específico. Observa-se que, para o caso em que possua quatro aceleradores e quatro módulos reconfiguráveis que poderão recebe-los, serão geradas 16 configurações diferentes, ou seja, 16 bitstreams parciais.

\section{EXEMPLO ILUSTRATIVO}

Seguiu-se a metodologia proposta para implementar uma simples calculadora de ponto flutuante de precisão simples seguindo o padrão IEEE-754 (IEEE, 2008), onde as operações de soma/subtração, multiplicação, divisão e raiz quadrada são implementadas em hardware. Utilizou-se da placa de desenvolvimento PYNQ-Z1, que possui o SoC Zynq-7000 fabricado pela Xilinx.

Utilizou-se o framework de código aberto FloPoCo (de Dinechin and Pasca, 2011) para gerar as implementações das operações aritméticas em ponto flutuante, sendo elas encapsuladas no padrão definido. Os aceleradores para soma/subtração, multiplicação e divisão implementam, respectivamente, as operações $S=A+B, S=A * B$ e $S=\frac{A}{B}$, sendo operações com dois operandos, enquanto o acelerador para a raiz quadrada implementa a operação $S=\sqrt{A}$, utilizando apenas um operador. A operação de subtração utiliza o acelerador de soma, apenas invertendo o operando B. Assim, para os aceleradores com dois operandos definiu-se:

- $\left(08_{16}\right)$ Registrador Propósito Geral 0: Operando A

- $\left(0 C_{16}\right)$ Registrador Propósito Geral 1: Operando B

\section{- $\left(10_{16}\right)$ Registrador Propósito Geral 2: Resultado S}

Similarmente, para o que utiliza um operando, definiu-se:

- $\left(08_{16}\right)$ Registrador Propósito Geral 0: Operando A

- $\left(0 C_{16}\right)$ Registrador Propósito Geral 1: Resultado S

Após a descrição e encapsulamento, é feito a síntese dos aceleradores, obtendo se os valores de utilização de recursos de cada um observados na Tabela 1 .

Tabela 1. Recursos utilizados pelos aceleradores.

\begin{tabular}{ccccc} 
Acelerador & LUT & FF & LUTRAM & DSP \\
\hline Somador & 533 & 529 & 46 & 0 \\
Multiplicador & 287 & 314 & 0 & 2 \\
Divisor & 2054 & 873 & 37 & 0 \\
Raiz Quadrada & 533 & 824 & 28 & 0 \\
\hline
\end{tabular}

Para a arquitetura, optou-se por implementar apenas um módulo reconfigurável para dar suporte aos aceleradores implementados. Fez-se a síntese da região estática e partiuse para o floorplanning, definindo a região do módulo reconfigurável.

A definição da região do módulo reconfigurável considera os maiores valores de utilização de cada tipo de recurso, para que ela possa atender todos os acelerados. Pela Tabela 1, obtém-se os seguintes valores para a região: $L U T=$ 2054, $F F=873$, LUTRAM $=46$ e $D S P=2$.

Para o caso de quatro aceleradores e um módulo reconfigurável, quatro configurações são possíveis. Gera-se então as configurações e as mesmas são verificadas, validando a compatibilidade entre elas.

Validadas todas as configurações, são geradas as bitstreams, sendo:

- Quatro bitstreams totais, contendo a região estática combinada com cada um dos aceleradores.

- Quatro bitstreams parciais, uma contendo apenas os aceleradores individuais.

As bitstreams totais são utilizadas para fazer a configuração inicial da região estática e o acelerador inicial, podendo o usuário escolher qual acelerador é inicialmente configurado no módulo reconfigurável. As bitstreams parciais são utilizadas pela API para a troca dos aceleradores, reconfigurando apenas o módulo reconfigurável.

\section{CONCLUSÃO}

Este trabalho apresentou uma metodologia de desenvolvimento de aplicações de Fog-Computing na indústria 4.0 utilizando SoC-FPGAs e reconfiguração parcial e dinâmica. Essa abordagem permite que mais poder computacional seja disponibilizado nos edge-devices por meio de aceleradores de hardware ao mesmo tempo em que se reduz a dissipação de potência e o custo, uma vez que uma mesma plataforma pode ser empregada em diversas aplicações. Foram apresentadas padronizações na interface entre os aceleradores de hardware e o processador embarcado que facilitam a integração de novas funcionalidades. Uma API foi também descrita, permitindo que os desenvolvedores de software utilizassem os aceleradores de forma transparente, sem a necessidade de conhecimento 
prévio da comunicação com o barramento dos aceleradores ou de como eles são reconfigurados no FPGA. Durante o desenvolvimento, percebeu-se a importância do floor planning para a utilização eficiente da técnica, e que as ferramentas atuais ainda não dão suporte para um floorplanning automático, sendo ele feito manualmente pelos projetistas. Este é, sem dúvida, um ponto importante para trabalhos futuros. Adicionalmente, observou-se dois gargalos de desempenho causados pela utilização de DPR: 1) o tempo gasto na reconfiguração, que apesar de ser menor do que em reconfigurações totais ainda podem ser relevantes dependendo da aplicação; e 2) a fragmentação dos recursos de hardware. Apesar desses pontos, os ganhos de flexibilidade, desempenho e dissipação de potência justificam o emprego da técnica em aplicações sensíveis a latência. Como exemplo, pode-se citar aplicações de visão computacional que necessitem de analisar em tempo real imagens do fluxo de produção.

Como oportunidades para trabalhos futuros, pode-se apontar as seguintes pesquisas:

(1) Explorar outras políticas de escalonamento de recursos, buscando reduzir o impacto do efeito do tempo de reconfiguração na aplicação.

(2) Explorar modelos para otimização do floorplanning dos módulos reconfiguráveis, de forma a reduzir o efeito da fragmentação dos recursos.

(3) Desenvolver uma ferramenta para explorar os tradeoffs na partição entre funcionalidades de software e hardware do sistema.

\section{REFERÊNCIAS}

ARM (2013). Amba axi and ace protocol specification.

Choi, K. (2011). Coarse-grained reconfigurable array: Architecture and application mapping. IPSJ Transactions on System LSI Design Methodology, 4, 31-46. doi:10. 2197/ipsjtsldm.4.31.

Claus, C., Zeppenfeld, J., Müller, F., and Stechele, W. (2007). Using partial-run-time reconfigurable hardware to accelerate video processing in driver assistance system. In Proceedings of the Conference on Design, Automation and Test in Europe, DATE '07, 498-503. EDA Consortium, San Jose, CA, USA. URL http://dl-acm-org.ez27.periodicos. capes.gov.br/citation. cfm?id=1266366. 1266473.

de Dinechin, F. and Pasca, B. (2011). Designing custom arithmetic data paths with flopoco. IEEE Design Test of Computers, 28(4), 18-27. doi:10.1109/MDT.2011.44.

IEEE (2008). Ieee standard for floating-point arithmetic. IEEE Std 754-2008, 1-70. doi:10.1109/IEEESTD.2008. 4610935.

Kareemullah, H., Janakiraman, N., and Kumar, P.N. (2017). A survey on embedded reconfigurable architectures. In 2017 International Conference on Communication and Signal Processing (ICCSP), 1500-1504. doi: 10.1109/ICCSP.2017.8286636.

Kelm, J.H. and Lumetta, S.S. (2008). Hybridos: Runtime support for reconfigurable accelerators. In Proceedings of the 16th International ACM/SIGDA Symposium on Field Programmable Gate Arrays, FPGA '08, 212-221. ACM, New York, NY, USA. doi:10. 1145/1344671.1344703. URL http://doi .acm.org/10. 1145/1344671.1344703.
Krill, B., Ahmad, A., Amira, A., and Rabah, H. (2010). An efficient fpga-based dynamic partial reconfiguration design flow and environment for image and signal processing ip cores. Signal Processing: Image Communication, 25(5), 377 - 387. doi:https://doi.org/10.1016/j. image.2010.04.005. URL http://www. sciencedirect. com/science/article/pii/S0923596510000494. Special Issue on Breakthrough Hardware Architectures.

Lie, W. and Feng-yan, W. (2009). Dynamic partial reconfiguration in fpgas. In 2009 Third International Symposium on Intelligent Information Technology Application, volume 2, 445-448. doi:10.1109/IITA.2009.334.

Mouradian, C., Naboulsi, D., Yangui, S., Glitho, R.H., Morrow, M.J., and Polakos, P.A. (2018). A comprehensive survey on fog computing: State-of-the-art and research challenges. IEEE Communications Surveys Tutorials, 20(1), 416-464. doi:10.1109/COMST.2017.2771153.

Nava, F., Sciuto, D., Santambrogio, M.D., Herbrechtsmeier, S., Porrmann, M., Witkowski, U., and Rueckert, U. (2011). Applying dynamic reconfiguration in the mobile robotics domain: A case study on computer vision algorithms. ACM Trans. Reconfigurable Technol. Syst., 4(3), 29:1-29:22. doi:10.1145/2000832.2000841. URL http://doi-acm-org.ez27.periodicos.capes. gov.br/10.1145/2000832.2000841.

Nguyen, M. and C. Hoe, J. (2018). Time-shared execution of realtime computer vision pipelines by dynamic partial reconfiguration. In 2018 28th International Conference on Field Programmable Logic and Applications (FPL), 230-2304. doi:10.1109/FPL.2018.00046.

Vahid, F. and Givargis, T. (2001). Embedded System Design: A Unified Hardware/Software Introduction. John Wiley \& Sons, Inc., New York, NY, USA, 1st edition.

Vatsolakis, C. and Pnevmatikatos, D. (2017). Racos: Transparent access and virtualization of reconfigurable hardware accelerators. In 2017 International Conference on Embedded Computer Systems: Architectures, Modeling, and Simulation (SAMOS), 11-19. doi:10.1109/ SAMOS.2017.8344606.

Xilinx (2018). Vivado design suite user guide: Partial reconfiguration. 